\title{
Persistent Mullerian Duct Syndrome: A Case Report
}

\author{
Shabnam Izhar, Purnima Singh* and Kusum Gupta
}

Department of Pathology, Vivekananda Polyclinic Institute of Medical Science(VPIMS), Lucknow, India

\section{ABSTRACT}

Persistent Mullerian duct syndrome (PMDS) is usually an accidental finding either during orchidopexy or during routine inguinal hernia repair in male patients presenting with undescended testes.It is a rare form of internal male pseudohermaphroditism caused by defect in synthesis or action of Mullerian inhibiting factor due to which Mullerian derivatives such as uterus, fallopian tube and upper vagina are normally present in 46XY males. Here we present a case report of a 7 year old male presenting with bilateral undescended testes.

\section{Keywords: Mullerian Inhibiting Substances, Hernia Uteri Inguinale, Persistent Mullerian Duct Syndrome.}

\section{Introduction}

Persistent Mullerian duct syndrome (PMDS) is a rare disorder of sex development characterized by the persistent of Mullerian duct derivatives in a genotypic (46 XY) and phenotypic normally male. ${ }^{[1]}$ PMDS is transmitted in an autosomal recessive manner caused by mutation in the anti mullarian hormone gene or in the gene encoding the AMH receptor. ${ }^{1}$ Patients often present with cryptorchidism or an inguinal hernia. Presence of uterus in the hernial sac is known as hernia uterine inguinale. ${ }^{[2]}$

\section{Case report}

A 7-year old child (reared as male) presented with swelling and pain with bilateral undescended testis. On clinical examination bilateral testes were not palpable. He had a history of testis not present in scrotum since birth. USG report showed right testis located in right inguinal canal near deep ring and left testis located in left inguinal canal near the superficial ring. The patient had hernia at the age of 1.5 years for which herniotomy was performed. On exploration, gonads were found in the hernial sac. The patient was planned, prepared and taken for laproscopic orchidopexy with excision of rudimentary organ. (Figure1) The specimen was sent to our department.

The gross examination showed a two brownish soft tissue piece together measuring $3.5 \mathrm{cms}$ in length. Cut section showed lumen. Microscopic evaluation showed finding of rudimentary Mullerian structure showing fallopian tube composed of numerous delicate plical folds with intact lumen. (Figure2, 3) Also identified few endometrial glands embedded in stroma. (Figure 4) Subsequently the condition was explained to the parents and karyotyping was done which was suggestive of 46XY male karyotype.

\section{Discussion}

The exact cause of PMDS is not known, however it is thought to result from absence of anti mullerian hormones (AMH ), abnormal AMH or defects in its receptor. ${ }^{[3]}$ The $\mathrm{AMH}$ (also known as Mullerian inhibiting substance) is a glycoprotein secreted by fetal sertoli cells. At 7 weeks of gestation, both the Mullerian ( paramesonephric ) and Wolffian (mesonephric ) ducts are present in a fetus. In male fetus (XY) Leydig cells secrete testosterone which has a local effect on Wolffian duct and help in differentiation into epididymis, vas deferens and seminal vesicle whereas dihydrotestosterone help in the formation of urogenital sinus and external genitalia. Another important function of MIS is to initiate testicular descent, principally by its postulated regulatory control over the gubernaculum testis. [4] These two functions of the MIS explain the clinical findings of PMDS.

There are three anatomic variants:

1. In the most common male type, one testis is usually found within the scrotum; the uterus and ipsilateral fallopian tube are either in the inguinal canal or can be brought into it by gentle traction on the presenting testis ${ }^{[5]}$.

2. In some cases, the contralateral testis and tube are also in the hernial sac; transverse testicular ectopia can also occur ${ }^{[6]}$.

3. The least common form or female type is characterized by bilateral cryptorchidism with testes embedded in the broad ligaments in an ovarian position with respect to the uterus which is fixed in the pelvis ${ }^{[7]}$.

For optimum management surgeon should be aware of this entity and recognition during operation (as most of the 


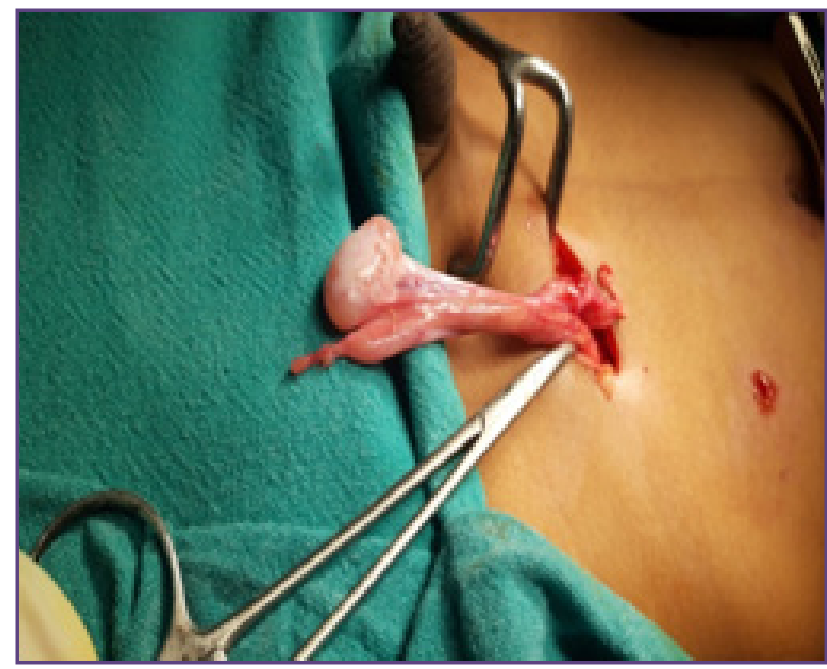

Fig. 1: Gross photograph of the abdominal mass.

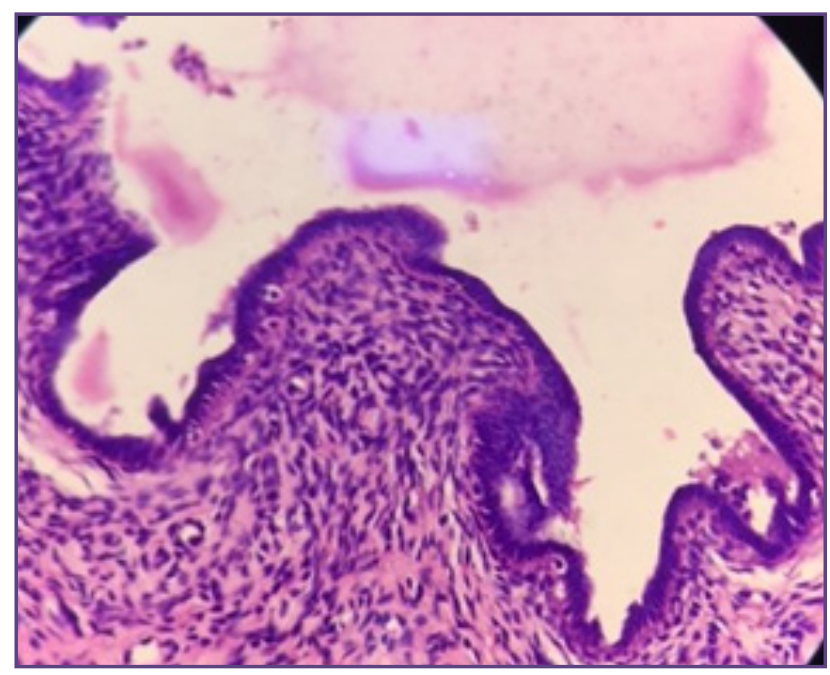

Fig. 3: 40x (H\&E) Plicae of fallopian tube lined by ciliated tall columnar epithelium.

time not suspected preoperatively) is most important. As in this case the diagnosis is often made incidentally during surgery for an inguinal hernia or during surgery exploration for cryptorchidism. Once suspected the confirmation of diagnosis is done with biopsies and chromosomal studies. After confirmation of the diagnosis definitive surgery consists of removal of the Mullerian remnants with orchidopexy or orchidectomy. Differential diagnosis of PMDS includes disorders of sexual differentiation with defective regression of the müllerian derivatives including mixed gonadal dysgenesis (MGD). Biopsy is required to confirm the sex and to differentiate from MGD. A workup to rule out associated genitourinary malformation should be done. In our case, the patients had no associated genitourinary malformation.

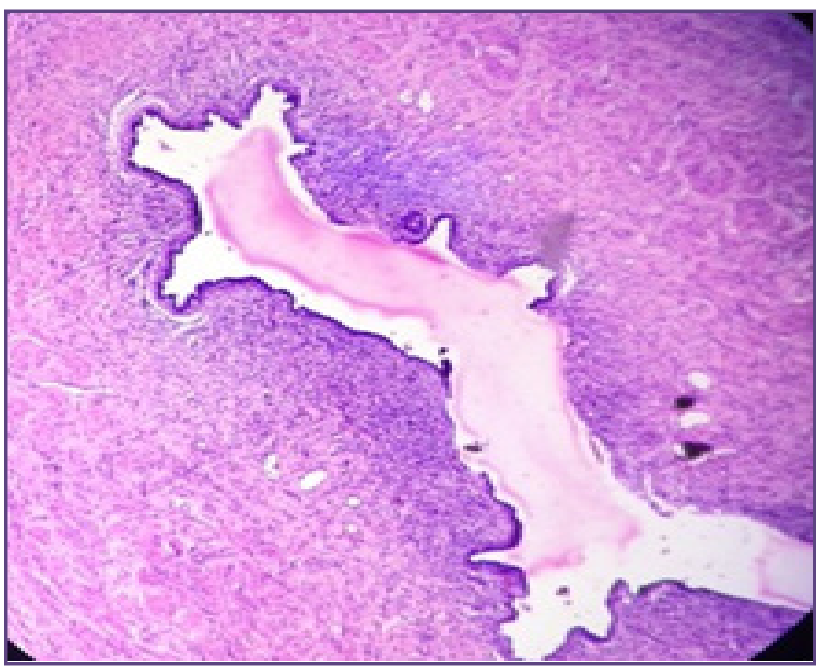

Fig. 2: 10x (H\&E) Photomicrograph showing fallopian tube with intact lumen.

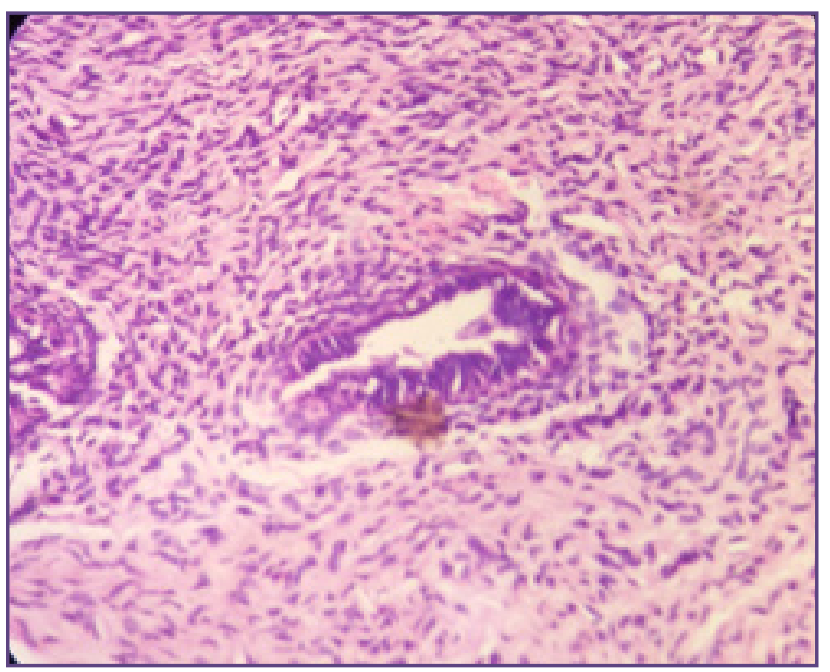

Fig. 4: 40x (H\&E) Photomicrograph showing endometrial glands and stroma.

Like other undescended testes, the gonads of these patients are at increased risk of malignant transformation. There have been case reports of embryonal carcinoma, seminoma, yolk sac tumor and teratoma in patients of PMDS. The overall incidence of malignant changes has been found to be $15 \%{ }^{[8]}$ In our case, neither testicular tissue nor any malignancy was identified in the multiple sections from the mass removed.

\section{Conclusion}

PMDS has an autosomal recessive inheritance. Screening of siblings and second degree relatives is necessary. A clinically challenging case of persistent mullerian duct syndrome required multidisciplinary team approach with aim to create awareness about this rare entity. The 
clinicians and the pathologists should be aware of the entity of PMDS while dealing with the cryptorchid patients. This is necessary to reduce and foresee complications like infertility and neoplastic transformation.

\section{References}

1. Gujar N, Choudhari R, Choudhari G et al. Male form of persistent Mullerian duct syndrome type1 (hernia uteri inguinalis) presenting as an obstructed inguinal hernia: a case report. Journal of medical case reports.2011; 5: 586.

2. Patil V, Muktinaini S, Patil R. Persistent Mullerian Duct Syndrome: a case Report. Indian J Surg. 2013; 75: 460-462.

3. Rey RA, Grinspon RP. Normal male sexual differentiation and etiology of disorders of sex development. Best Pract Res Clin Endocrinol Metab 2011; 25:221-38.
4. Robboy SJ, Bentley RC, Russell P et al. Pathology of abnormal sexual development. Obstetrical and Gynecological Pathology.5th ed. United Kingdom: Churchill Livingstone Elsvier. 2003; 1209-32.

5. Di Clemente N, Belville C. Anti-Mullerian hormone receptor defect. Best Pract Res Clin Endocrinol Metab.2006; 20: 599610

6. Boleken ME, Kaya M, Guran S et al. Persistent Mullerian duct syndrome with transverse testicular ectopia. Int Urol Nephrol.2007;39(4):1173-1175

7. David AD. Sexual differentiation: normal and abnormal. In: Wein AJ, Kavoussi LR, Novick AC, Partin AW, Peters CA, editors. Campbell-Walsh urology. 9th ed. Philadelphia: Saunders; 2007: 3826-3827.

8. Dekker HM, De Jong IJ, Sanders J et al. Persistent Mullerian Duct Syndrome. Radiographics. 2003; 23:309-13.

*Corresponding author:

Purnima Singh, Department Of Pathology, Vivekananda Polyclinic Institute of Medical science, Niralanagar, Lucknow 226007 INDIA

Phone: +91 7985119182

Email: ps2545@gmail.com

Financial or other Competing Interests: None. 